\title{
Cardiomiopatia de Takotsubo como Causa de Infarto Agudo do Miocárdio em Paciente em Uso de Anti-Histamínico Associado a Pseudo-Efedrina
}

\author{
José Armando Mangione', Gustavo Ithamar Souto Maior ${ }^{1}$
}

\section{RESUMO}

Relatamos o caso de uma senhora de 77 anos, que vinha em uso de anti-histamínico associado a pseudo-efedrina por processo alérgico de vias aéreas superiores, admitida no Pronto Socorro com quadro de infarto agudo do miocárdio com elevação do segmento ST em parede ântero-lateral. O cateterismo cardíaco revelou artérias coronárias tortuosas e sem lesões obstrutivas, com hipercontratilidade basal associada à acinesia ântero-apical à ventriculografia esquerda. Devido a derrames pericárdicos de repetição, foi submetida à drenagem pleuro-pericárdica. Causas reumatológicas foram excluídas. Após 30 dias, recebeu alta em boas condições clínicas.

DESCRITORES: Infarto do miocárdio. Antagonistas dos receptores $\mathrm{H} 1$ de histamina. Cardiomiopatias, fisiopatologia. Cardiomiopatias, etiologia. Efedrina, efeitos adversos.

\section{SUMMARY}

Acute Myocardial Infarction Resulting from Takotsubo Cardiomyopathy in Patient on Antihistaminics Associated to Pseudoephedrin

This is the report on a 77-year-old female patient on antihistaminic associated to pseudoephedrin to treat an allergy condition in upper airways. The patient was admitted to the Emergency Unit with acute myocardial infarction and ST segment elevation in the antero-lateral wall. Cardiac catheterism showed tortuous coronaries, no obstructive lesions, and baseline hypercontractility associated to antero-apical akinesia on left ventriculography. As a result of repeated pericardial strokes, the patient was submitted to pleuro-pericardial drainage. Rheumatological causes were ruled out. The patient was discharged after 30 days in good clinical conditions.

DESCRIPTORS: Myocardial infarction. Histamine H1 antagonists. Cardiomyopathies, physiopathology. Cardiomyopathies, etiology. Ephedrine, adverse effects.
$\mathbf{R}$ elatamos o caso de uma paciente admitida no Pronto Socorro com quadro clínico compatível com infarto agudo do miocárdio (IAM): dor torácica típica, elevação do segmento ST (ESST) ao eletrocardiograma e alteração sérica de enzimas cardíacas, diagnosticando-se, posteriormente, Síndrome de Takotsubo (artérias coronárias sem obstruções e ventriculografia revelando ventrículo esquerdo com aspecto de "haltere": acinesia apical e hipercontratilidade basal) desencadeada, possivelmente, pelo uso da associação de pseudo-efedrina e anti-histamínico oral.

\section{RELATO DO CASO}

A paciente R.M.T, japonesa, de 77 anos, foi admi-

\footnotetext{
Hospital Beneficência Portuguesa de São Paulo, SP. Correspondência: Gustavo I. Souto Maior. Hospital Beneficência Portuguesa de São Paulo. Rua Maestro Cardim, 769 - 10 SS - Sala 71 - Bloco 1 - São Paulo - SP - Brasil - CEP 01323-900 - Tel: (11) 3505-1000 Fax: (11) 3251-2528 - 3284-6803 - E-mail: gustavo.maior@uol.com.br Recebido em: 10/12/2006 • Aceito em: 13/2/2007
}

tida, em 28 de agosto de 2006, no Pronto Socorro (PS), com precordialgia opressiva há aproximadamente 40 minutos, de forte intensidade, associada a sinais neurovegetativos. Tinha antecedentes de dislipidemia e fazia, há pouco mais de cinco dias, uso de anti-histamínico à base de loratadina associado a pseudo-efedrina, devido a processo alérgico de vias aéreas superiores.

Ao exame clínico cardiovascular, evidenciamos ritmo cardíaco regular em dois tempos, com bulhas cardíacas normofonéticas e ausência de sopros, frêmitos ou edema de membros inferiores. A ausculta pulmonar não apresentava alterações significativas. Os pulsos periféricos eram simétricos e as extremidades aquecidas e bem perfundidas. A pressão arterial era de 130/70 $\mathrm{mmHg}$, freqüência cardíaca de 94 bpm, freqüência respiratória de 22 ipm e temperatura axilar de $37^{\circ} \mathrm{C}$. Eletrocardiograma de 12 derivações revelou ESST de 3mm, em V3,V4, V5, V6 e de $2 \mathrm{~mm}$ em DIII e AVF (Figura 1).

Frente ao quadro clínico compatível com IAM com ESST, Killip \& Kimball I' , a paciente foi medicada 
Mangione JA, Maior GIS. Cardiomiopatia de Takotsubo como Causa de Infarto Agudo do Miocárdio em Paciente em Uso de AntiHistamínico Associado a Pseudo-Efedrina. Rev Bras Cardiol Invas 2007; 15(1): 73-76.

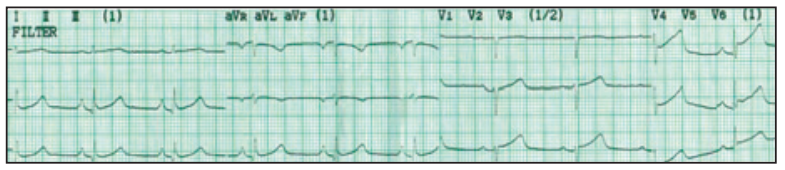

Figura 1 - Eletrocardiograma de 12 derivações - presença de elevação do segmento ST de $2 \mathrm{~mm}$ de $\mathrm{V} 3$ a V6 e de $1 \mathrm{~mm}$ em parede inferior, associado à amputação de onda $R$ de $V 1$ a V3.

com 300mg de aspirina, 600mg de clopidogrel e encaminhada à sala de hemodinâmica, ainda com precordialgia, 30 minutos após sua chegada ao PS. A cinecoronariografia evidenciou artérias tortuosas e isentas de obstruções (Figuras 2 e 3). Entretanto, os níveis de troponina e CKMB massa de admissão foram, respectivamente, $0,723 \mathrm{ng} / \mathrm{ml}(<0,01 \mathrm{ng} / \mathrm{ml})$ e $8,75 \mathrm{ng} / \mathrm{ml}$ $(<5,0 \mathrm{ng} / \mathrm{ml})$. À ventriculografia esquerda (Figuras $4 \mathrm{e}$ 5), observou-se hipercontratilidade basal associada a acinesia ântero-apical, com o ventrículo esquerdo (VE) apresentando aspecto de moringa ou haltere, compatível com a Síndrome de Takotsubo (ST) ${ }^{2,3}$. A pressão diastólica final do VE verificada foi de $20 \mathrm{mmHg}$.

Após a realização do estudo hemodinâmico, a paciente foi encaminhada à Unidade Coronariana (UCO) e submetida à realização de um ecocardiograma transtorácico (ETT), que corroborou com o diagnóstico da ST ao revelar VE com acinesia médio-apical ânteroseptal e fração de ejeção de 0,65 Simpson, às custas

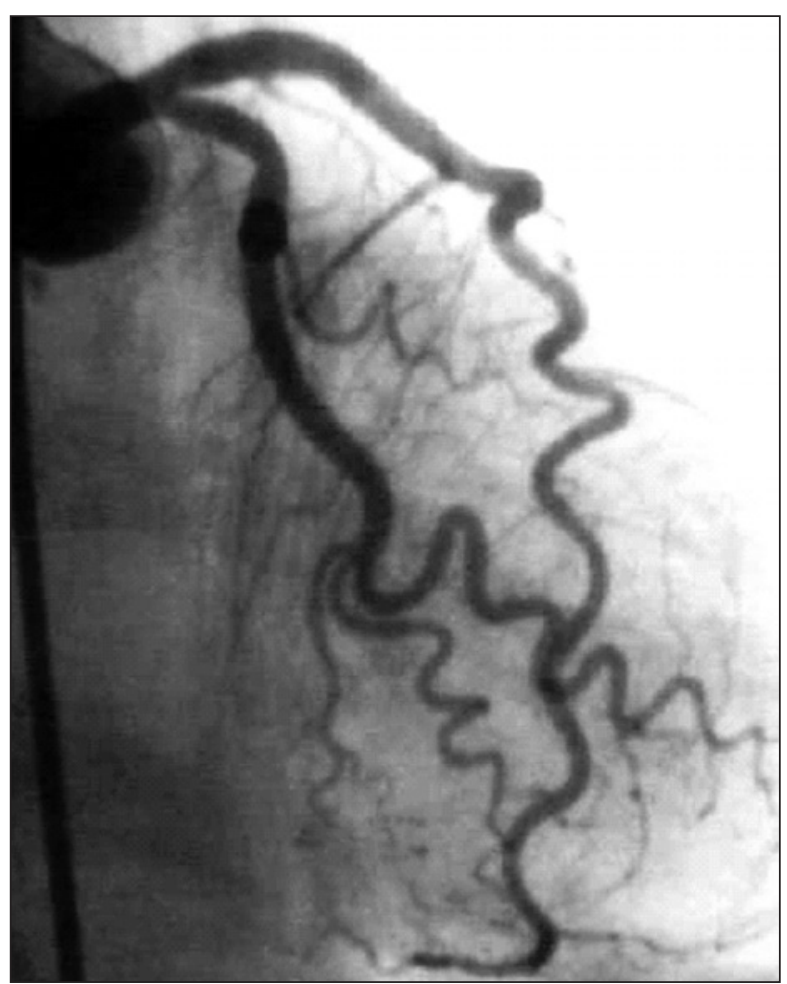

Figura 2 - Coronariografia esquerda: artéria coronária esquerda tortuosa visualizada em projeção ântero-posterior cranial. de uma hipercontratilidade das porções basais, determinando gradiente intraventricular de $15 \mathrm{mmHg}$. Não se evidenciou sinais de derrame pericárdico.

Em 30 de agosto, já na enfermaria e em uso de carvedilol 6,25 mg ao dia, a paciente apresentou novo episódio de dor torácica, desta vez em hemitórax direito, de forte intensidade e início súbito, com irradiação para região infra-escapular direita, associada a náuseas e sudorese, que não piorava aos movimentos ou à palpação. Diante de um possível evento tromboembólico pulmonar (TEP), solicitou-se dosagem de D-dímero, que veio discretamente aumentado, ultra-sonografia com doppler de membros inferiores, que excluiu trom-

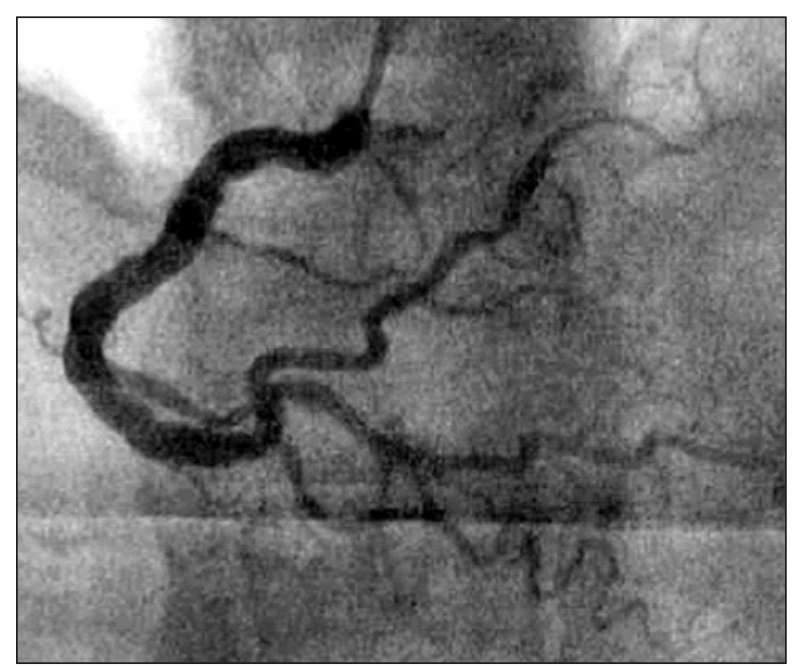

Figura 3 - Coronariografia direita: artéria coronária direita visualizada em projeção ântero-posterior cranial.

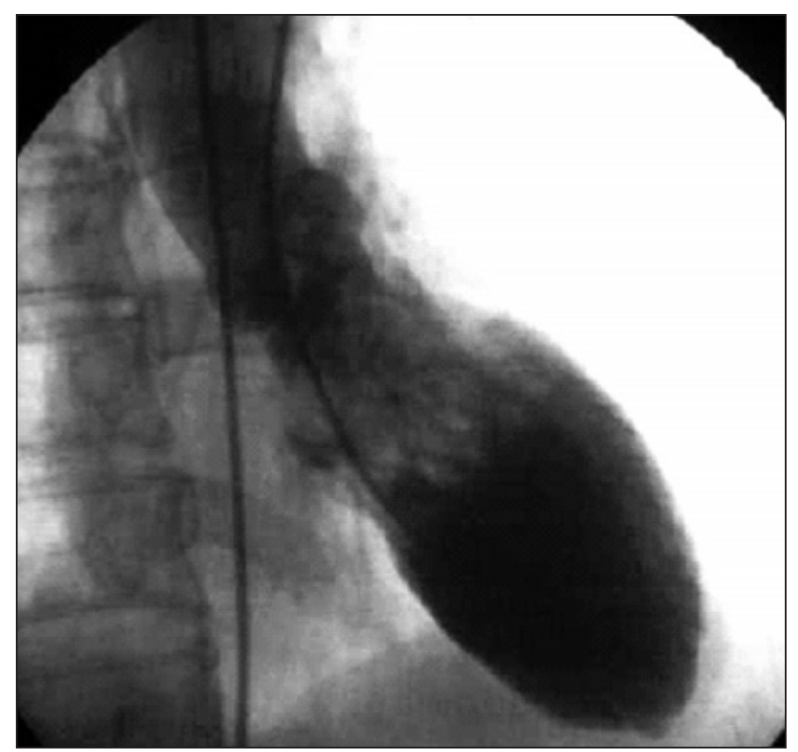

Figura 4 - Ventriculografia esquerda: ventrículo esquerdo em diástole máxima visualizado em projeção oblíqua anterior direita. 

Histamínico Associado a Pseudo-Efedrina. Rev Bras Cardiol Invas 2007; 15(1): 73-76.

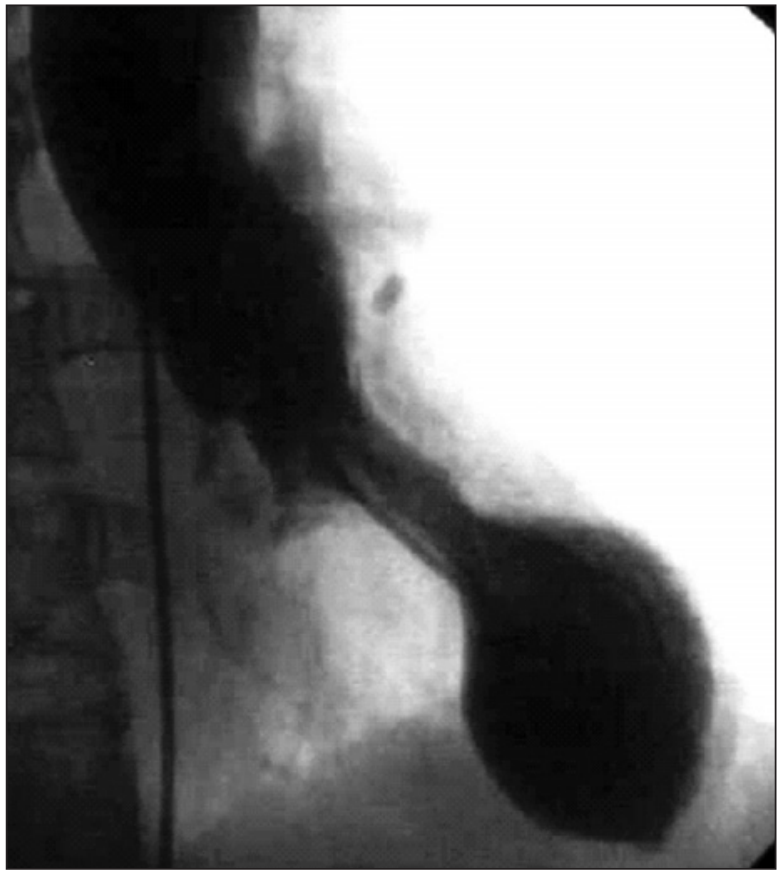

Figura 5 - Ventriculografia esquerda: ventrículo esquerdo em sístole, visualizado em projeção oblíqua anterior direita, com acinesia apical e hipercontratilidade das regiões basais, configurando um aspecto de haltere.

bose venosa profunda (TVP) e tomografia computadorizada helicoidal de tórax, negativo para TEP de acordo com o protocolo específico, revelando, no entanto, derrame pericárdico discreto/moderado. A dor torácica foi aliviada com analgésico opióide oral, contudo, a paciente evoluiu com dispnéia progressiva e cansaço.

Em 4 de setembro, novo ETT revelou VE com tamanho e função preservados, hipocinesia apical ânteroseptal, fração de ejeção de 0,76 , pressão sistólica de ventrículo direito de $32 \mathrm{mmHg}$ e um derrame pericárdico moderado a importante. O ecocardiograma transesofágico acrescentou ateromatose discreta e excluiu a possibilidade de dissecção de aorta. Neste mesmo dia, a paciente foi encaminhada à Unidade de Terapia Intensiva e submetida à punção pericárdica, com retirada de $210 \mathrm{ml}$ de líquido sero-sanguinolento, cuja análise bioquímica e onco-citológica mostrou: albumina $0,6 \mathrm{mg} / \mathrm{dl}$; glicose $21 \mathrm{mg} / \mathrm{dl}$; com ausência de presença de células neoplásicas. O perfil de coagulação da paciente era normal. Após estabilização clínica, a paciente recebeu alta para a enfermaria no dia seguinte.

Cinco dias após, a dor e a dispnéia recorreram e novo ETT revelou derrame pericárdico importante, desta vez, com sinais de restrição de câmaras direitas e derrame pleural esquerdo moderado. Foi, então, readmitida na UTI e submetida à drenagem pleuro-pericárdica com biópsia do pericárdio. Frente à poliserosite e para pesquisa de uma provável colagenose como etiologia subjacente ao quadro clínico, foi solicitado acompanha- mento especializado da Reumatologia. As seguintes provas laboratoriais foram negativas: FAN, anti-Ro, anti-La, p-ANCA, c-ANCA, anti-DNA, anti-cardiolipina IgG e IgM. A análise histopatológica do fragmento de pericárdio biopsiado mostrou processo inflamatório inespecífico.

Em 15 de setembro, um episódio de taquicardia supraventricular foi revertido com metoprolol endovenoso. Posteriormente, a paciente permaneceu em observação na enfermaria até o dia 28 de setembro, quando recebeu alta hospitalar em boas condições clínicas, dois dias após a retirada dos drenos pericárdico e pleural, com contração segmentar preservada, segundo ETT realizado no dia anterior.

\section{DISCUSSÃO}

O número de pacientes com dor torácica admitidos nas salas de emergência cresce cada vez mais à medida que as melhores condições de vida da população proporcionam uma maior longevidade.

Embora a mortalidade hospitalar do IAM tenha sido reduzida de 30\%, na década de 50, para os atuais 6 a $10 \%{ }^{4}$, o diagnóstico precoce desta entidade clínica permanece como uma das maiores preocupações médicas, procurando-se obter a patência da artéria culpada de forma precoce por meio da terapia de reperfusão mecânica ou química.

Particularmente em pacientes acima de 65 anos de idade, o IAM é responsável por mais de $80 \%$ dos óbitos, fazendo com que este quadro clínico seja a principal suspeita diagnóstica frente a um paciente com dor torácica associada a alterações enzimáticas e eletrocardiográficas.

A doença aterosclerótica é a principal etiologia de IAM, entretanto, outras causas raras, mas não tão infreqüentes, devem ser lembradas. Relatamos, neste artigo, um caso de ST em paciente idosa, provavelmente, precipitada pelo uso de pseudo-efedrina, levando a quadro clínico compatível com IAM.

A ST é também conhecida como Cardiomiopatia de Takotsubo, Síndrome do Coração Partido (Broken Heart Syndrome), Síndrome do Balonamento Apical Ventricular Esquerdo ou Cardiomiopatia do Estresse Humano (Human Stress Cardiomiopathy) ${ }^{5}$. Semanticamente, a palavra japonesa takotsubo é utilizada devido à semelhança do VE, na angiografia contrastada, com uma armadilha de pescar polvo.

Trata-se de uma disfunção ventricular esquerda transitória, inicialmente descrita no Japão ${ }^{5}$, precipitada, na maioria das vezes, por estresse físico ou emocional ${ }^{6,7}$, que se manifesta sob a forma de dor torácica associada a alterações eletrocardiográficas e elevação de enzimas cardíacas compatíveis com IAM, porém sem obstruções coronarianas significativas.

Os pacientes mais acometidos são do sexo femi- 
Mangione JA, Maior GIS. Cardiomiopatia de Takotsubo como Causa de Infarto Agudo do Miocárdio em Paciente em Uso de AntiHistamínico Associado a Pseudo-Efedrina. Rev Bras Cardiol Invas 2007; 15(1): 73-76.

nino em uma proporção de, aproximadamente, 6,3:1 e, normalmente, com idade superior a 65 anos. A disfunção ventricular é transitória, observando-se reversão muitas vezes completa por volta do $20^{\circ}$ dia, ao contrário das suas manifestações clínicas que geralmente persistem por um período um pouco mais prolongado.

A causa desta discinesia apical transitória ainda é uma incógnita. Sua incidência também é desconhecida, mas parece ser mais comum do que se pensa. Provavelmente, esta síndrome ainda é uma causa de atordoamento ventricular pouco diagnosticada.

Acredita-se que um dentre três fatores possa precipitar a ST: isquemia secundária a vasoespasmo epicárdico associado ao aumento do tônus simpático; espasmo da microcirculação e injúria miocárdica direta mediada por catecolaminas.

Embora cada vez mais freqüentes, ainda são escassas as publicações a respeito da ST na literatura ocidental e, em particular, no Brasil. Vasconcelos et al. ${ }^{8}$ relataram um caso de uma paciente com ST que evoluiu com choque cardiogênico. Mais recentemente, Meyer Neto et al. ${ }^{9}$ publicaram um caso da associação da ST com obesidade mórbida e lúpus eritematoso sistêmico.

Bybee et al. ${ }^{10}$, em acompanhamento de cinco pacientes com ST, verificaram que as mulheres pós-menopausa (62 a 75 anos) foram mais acometidas. Estes dados são concordantes com a série de Akashi et al. ${ }^{11}$, que acompanhou sete pacientes, dos quais seis eram mulheres idosas e apenas um do sexo masculino (idade média de 75,3 anos). Todos os pacientes tinham sido submetidos a importante estresse emocional. As enzimas cardíacas não apresentaram aumento significativo, ao contrário dos níveis séricos de epinefrina $(1,19 \mathrm{ng} / \mathrm{ml})$. A função ventricular se normalizou em média em 17,4 dias, em cinco pacientes e suporte hemodinâmico por três dias foi necessário nos outros dois.

A excepcionalidade do caso clínico relatado devese não apenas à raridade da ST, mas também ao fato do evento ter sido, provavelmente, desencadeado por um estresse medicamentoso por meio da atividade adrenérgica da pseudo-efedrina. Ressaltamos, ainda, que na literatura existem relatos de ruptura ventricular seguida de choque cardiogênico, todavia, não há referência de uma evolução clínica com derrame pericárdico de repetição.

O quadro clínico de apresentação está de acordo com a literatura no que diz respeito a idade, sexo e forma de apresentação. É importante salientar que a alteração eletrocardiográfica encontrada foi uma ESST, enquanto na literatura a presença de ondas $T$ negativas e gigantes (ondas T cerebrais), semelhantes às encontradas nos casos de hemorragia subaracnóidea, com infradesnivelamento do segmento ST associado ou não ao prolongamento do intervalo QT são achados mais comuns ${ }^{12}$.

A hipótese de colagenose desencadeando vasculite e conseqüente síndrome coronariana aguda foi aventa- da, porém excluída diante das provas laboratoriais reumatológicas negativas (FAN, anti-Ro, anti-La, p-ANCA, c-ANCA, anti-DNA, anti-cardiolipina $\lg G$ e $\lg M)$ e da evolução clínica favorável da paciente na ausência de antiinflamatórios, tanto não esteroidais quanto esteroidais.

Este relato demonstra, ainda, a importância do estudo hemodinâmico no diagnóstico etiológico da síndrome coronariana aguda. A exclusão da coronariopatia obstrutiva aterosclerótica evita que muitos pacientes com ST possam apresentar as complicações hemorrágicas decorrentes do uso inadvertido dos agentes fibrinolíticos.

Um quadro semelhante ao IAM, numa paciente idosa, do sexo feminino, pode ser decorrente da ST. A ausência de obstruções à cinecoronariografia, a reversibilidade da disfunção ventricular e o achado típico do balonamento apical à ventriculografia esquerda selam o diagnóstico desta forma de atordoamento miocárdico.

\section{REFERÊNCIAS BIBLIOGRÁFICAS}

1. Killip T 3rd, Kimball JT. Treatment of myocardial infarction in a coronary care unit: a two year experience with 250 patients. Am J Cardiol 1967;20:457-64.

2. Kawai S, Suzuki H, Yamaguchi H, Tanaka K, Sawada H, Aizawa T, et al. Ampulla cardiomyopathy ("Takotsubo cardiomyopathy") - reversible left ventricular dysfunction: with ST segment elevation. Jpn Circ J 2000;64:156-9.

3. Satoh H, Tateishi H, Uchida T. Takotsubo-type cardiomyopathy due to multivessel spasm. In: Kodama K, Haze K, Hon M, eds. Clinical aspects of myocardial injury: from ischemia to heart failure. Tokyo: Kagakuhyouronsya;1990. p.56-64.

4. DATASUS - Departamento de Informática do SUS - Ministério da Saúde, Governo do Brasil. http: www.datasus.gov.br

5. Guttormsen B, Nee L, Makielski JC, Keevil JG. Transient left ventricular apical ballooning: a review of the literature. WMJ 2006;105:49-54

6. Tsuchihashi K, Ueshima K, Uchida T, Oh-mura N, Kimura $\mathrm{K}$, Owa $\mathrm{M}$, et al. Transient left ventricular apical ballooning without coronary artery stenosis: a novel heart syndrome mimicking acute myocardial infarction. J Am Coll Cardiol 2001;38:11-8.

7. Kurisu S, Sato H, Kawagoe T, Ishihara M, Shimatani Y, Nishioka K, et al. Tako-tsubo-like left ventricular dysfunction with ST-segment elevation: a novel cardiac syndrome mimicking acute myocardial infarction. Am Heart J 2002; 143:448-55.

8. Vasconcelos JT, Martins S, Sousa JF, Portela A. Cardiomiopatia de Takotsubo: uma causa rara de choque cardiogênico simulando infarto agudo do miocárdio. Arq Bras Cardiol 2005;85:128-30.

9. Meyer Neto JG, Benchimol CB, Penna GL, Carvalho LA, Pessoa MC. Associação rara entre lúpus eritematoso sistêmico, obesidade mórbida e síndrome de Takotsubo. Arq Bras Cardiol 2006;87:116-21.

10. Bybee KA, Kara T, Prasad A, Lerman A, Barsness GW, Wright RS, et al. Systematic review: transient left ventricular apical ballooning: a syndrome that mimics ST-segment elevation myocardial infarction. Ann Intern Med 2004;141:858-65.

11. Akashi YJ, Nakazawa K, Sakakibara M, Miyake F, Koike H, Sasaka K. The clinical features of takotsubo cardiomyopathy. QJM 2003;96:563-73.

12. Wittstein IS, Thiemann DR, Lima JA, Baughman KL, Schulman SP, Gerstenblith G, et al. Neurohumoral features of myocardial stunning due to sudden emotional stress. N Engl J Med 2005;352:539-48. 